\title{
Long lasting response of trabectedin in patient with gastric leiomyosarcoma with liver metastasis: an update to previous report
}

\section{Future Science}

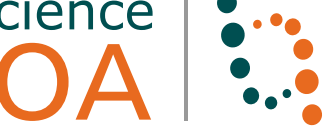

\author{
Sameer Rastogi ${ }^{* *, 1}$, Kaushal Kalra ${ }^{1}$, Parisa Manasa2 ${ }^{2}$ Monali Rajawat ${ }^{3,4}$ \& Varshil \\ Mehta*,4,5 iD \\ ${ }^{1}$ Department of Medical Oncology, All India Institute of Medical Sciences, New Delhi 110029, India \\ ${ }^{2}$ Sachin Sarcoma Society, New Delhi 110036, India \\ ${ }^{3}$ Sheffield Teaching Hospitals NHS Foundation Trust, Northern General Hospital, Sheffield S5 7AU, UK \\ ${ }^{4}$ Medkrux Research Group, Mumbai 400064, India \\ ${ }^{5}$ Chelsea and Westminster Hospital NHS Foundation Trust, West Middlesex University Hospital, Isleworth TW7 6AF, UK \\ *Author for correspondence: varshil.mehta@chelwest.nhs.uk \\ **Author for correspondence: samdoc_mamc@yahoo.com
}

Leiomyosarcoma of the stomach is an extremely rare malignancy for which treatment in advanced disease is hardly reported. Here, we report a case of a 48-year-old man with metastatic gastric Leiomyosarcoma who had previously received a combination of gemcitabine and docetaxel followed by pazopanib after detection of metastasis. The patient was started on trabectedin as per protocol and had disease control continuing for 17 cycles of trabectedin. His quality of life and absence of significant toxicities highlight the noncumulative nature of trabectedin and potential benefit in responding cases.

Lay abstract: Leiomyosarcoma (LMS) is a very rare form of tumor which, when it spreads to other organs, can be dreadful. Here, we report a case of a 48-year-old man who was diagnosed with LMS of the stomach that subsequently affected the liver. Multiple lines of anti-cancer drugs were given to the patient. At present, the patient takes trabectedin and has been tolerating it very well. Most drugs have cumulative toxicity when given for a long period of time but trabectedin does not possess this effect, which is why we feel that trabectedin should be considered as first-line treatment for LMS in the future.

First draft submitted: 18 July 2019; Accepted for publication: 30 September 2019; Published online: 9 December 2019

Keywords: gastric LMS • liposarcoma $\bullet$ LMS • metastasis • pazopanib • trabectedin

In the last few years, the landscape of sarcoma management has changed with the approval of new and better agents. After pazopanib was approved in nonadipocytic sarcomas, trabectedin received US FDA approval in October, 2015 for the treatment of advanced L-sarcomas (liposarcoma and leiomyosarcoma [LMS]) in patients previously treated with anthracyclines. LMS is one of the most common soft tissue sarcomas and arises from smooth muscle cells. Both the approval trials of pazopanib and trabectedin had LMS as the most common subtype $[1,2]$.

Patients treated with trabectedin had longer progression-free survival (PFS) than patients who received dacarbazine (4.2 vs 1.5 months; $\mathrm{p}=0.001)$. However, there was no difference in overall survival (OS) in the two arms [2].

Trabectedin has a pleiotropic mechanism of action including the interaction with the minor groove of the DNA double helix, affecting transcription of different genes involved in DNA repair, facilitating lethal DNA strand breaks, directing growth inhibition and resulting in the death of malignant cells. It also displays anti-inflammatory and immunomodulatory properties because of the hinderance of factors that promote tumor growth, angiogenesis and metastasis $[3,4]$. Some of the most common adverse effects (all grades) of trabectedin in registration trials were nausea $(73 \%)$, fatigue (67\%), neutropenia (49\%), increased AAT (39\%), vomiting (44\%), anemia (39\%) etc [2].

Here, we report an unusual case of gastric LMS that had earlier responded to pazopanib [5] and as subsequent therapy had long-lasting response to trabectedin. 


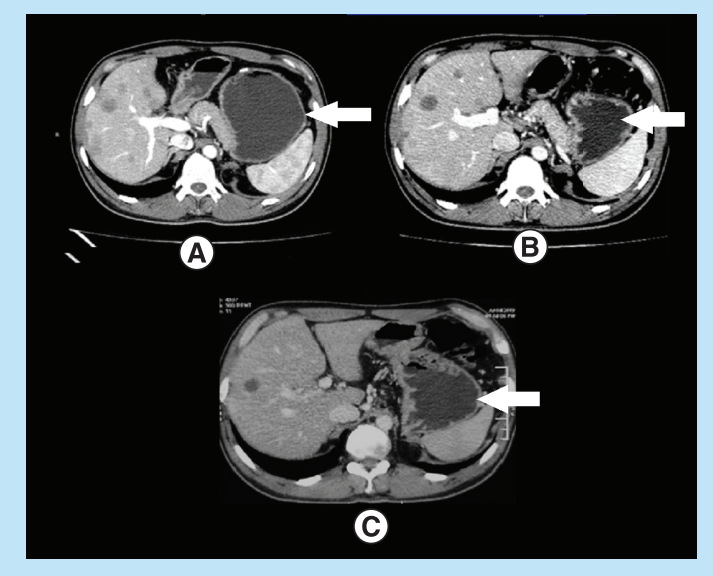

Figure 1. Computed tomography scan showing the largest lesion: (A) before start of the treatment; (B) post 3 cycles; (C) post 17 cycles.

\section{Case presentation}

A 48-year-old man having no co-morbidities was diagnosed with LMS of stomach (greater curvature) in his local area and underwent mass excision for the same in December, 2014. The histopathology was suggestive of high-grade LMS. The patient received adjuvant chemotherapy (6 cycles) of doxorubicin. After a treatment-free interval of 18 months, he developed metastasis to the liver in September, 2016. The patient further was given gemcitabinedocetaxel (7 cycles), after which the disease progressed. Subsequently, the patient came to our institute for further therapy. At presentation, he had Eastern Cooperative Oncology Group Performance Status (ECOG PS) 1 and we then started him on pazopanib $400 \mathrm{mg}$.

The patient had partial response initially on pazopanib (dosage: $400 \mathrm{mg} \mathrm{OD}$ ) but after 6 months of therapy, the disease progressed. Hence, he was started on injection trabectedin from February 2018 onwards. Trabectedin was given at the dose of $1.5 \mathrm{mg} / \mathrm{m} 2$ on every $21^{\text {st }}$ day (every 3 weeks). Post three cycles of trabectedin, a partial response was observed. After eight cycles, a stable disease was noted in imaging by RECIST 1.1 criteria. He tolerated trabectedin well and had fatigue as the only toxicity (Grade 1), while adverse reactions such as alopecia and mucositis never developed during the treatment. The last cycle of trabectedin was given in May 2019 (17th cycle) and the imaging scans showed stable disease. His current ECOG PS is 1 and doing well on therapy.

The computed tomography scan before initiation of the treatment displayed the largest lesion with the dimension of $30.0 \mathrm{~mm} \times 22.5 \mathrm{~mm}$ (Figure 1A), which subsequently reduced to $20 \mathrm{~mm} \times 10 \mathrm{~mm}$ and $16 \mathrm{~mm} \times 14 \mathrm{~mm}$ post 3 cycles (Figure 1B) and 17 cycles from the initiation of the therapy (Figure 1C) respectively. The disease is currently stable at 58 months after diagnosis of primary LMS was made.

\section{Discussion}

We here describe a very rare case of a patient with primary gastric LMS with secondary metastasis to liver, who was treated with multiple lines of chemotherapy. The gastric LMS is an extremely rare malignant tumor, comprising only $1 \%$ of all the gastric malignancies. In an analysis of the Surveillance, Epidemiology and End Results (SEER) database in patients with primary gastric LMS diagnosed between 1988 to 2008, the median OS of gastric LMS was significantly higher than gastric adenocarcinoma (36 vs 10 months; $\mathrm{p}=0.001)$ [6].

As mentioned in previous reports, the closest differential diagnosisCT of gastric LMS is gastrointestinal stromal tumor; however, the pathology and immunohistochemistry of our patient had been reviewed elaborately and was suggestive of LMS [5]. As this patient responded to a combination of gemcitabine-docetaxel, pazopanib and trabectedin, it is possible that the behavior of gastric LMS is similar to extremity LMS.

The patient has nonprogressive disease on 17 th cycle of trabectedin with preserved quality of life and minimal toxicities, underlining the potential benefit of trabectedin in metastatic LMS. The minimal toxicity profile in our patient further reinforces the fact that the toxicity of trabectedin is non-cumulative as has been shown in previous trials $[7-10]$.

In the trial by Demetri et al., it was reported that $10 \%$ of patients were able to take 12 cycles of trabectdine continuously as compared with $2 \%$ of patients which took dacarbazine, thus a considerably smaller number of patients continues beyond 12 cycles [1]. Tavella et al. reported a case of uterine LMS, in which the patient was 
treated with 30 cycles of trabectedin and had a stable disease till the case was reported [11]. Similarly, in a study by Nteli et al., the patient with uterine LMS had a stable disease till ninth cycle of chemotherapy [12]. In a report by Hauslbauer $e t$ al., trabectedin provided 22 months of progression-free time with good quality of life in an elderly man with LMS with multiple comorbidities [13]. In a report by Corrado et al., the best response to trabectedin happened after 9 cycles in a patient with metastatic inguinal LMS, signifying late response even after three to six cycles [14]. To our best knowledge there is no report of use of trabectedin in gastric LMS.

Since only a fraction of patients show clinical improvement after being exposed to trabectedin, it is imperative to use a biomarker in order to delineate the subgroup of the patients that will derive maximum benefit from the study. In a few clinical pharmacogenomic retrospective studies, it was shown that BRCA1 status could be predictive of trabectedin efficacy in sarcoma patients, which was later on refuted in the EORTC 62091 study in which prospective study of BRCA 1 assessment in soft tissue sarcoma was done (BRCA study) [15].

\section{Newer modalities of treatment options available for LMS}

In the last few years various drugs like olaratumab, eribulin and some immunotherapies have also been tried in LMS. Olaratumab was approved in October 2016 in first-line setting in combination with doxorubicin, based upon a Phase Ib/II trial showing the dramatic OS benefit of 11.8 months as compared with single agent doxorubicin, which was unprecedented, leading to both reassurance and speculations after FDA accelerated approval [16]. This was followed by a Phase III placebo control randomized trial (ANNOUNCE), which used OS as primary end point. However, this trial failed to show OS benefit, with OS 20.4 months in the olaratumab/doxorubicin arm as compared with 29.8 months in the doxorubicin/placebo arm [17].

In a Phase III trial by Schoffski $e t$ al., patients with advanced L-sarcomas who previously received two lines of chemotherapy were randomized to eribulin and dacarbazine [18]. The primary end point was OS while secondary end points were progression-free survival and progression-free rate. There was statistically significant improvement in OS in the eribulin arm as compared with that of darcarbazine in the overall population. As per preplanned subgroup analysis, the benefit was mainly limited to the liposarcoma subgroup as compared with the LMS subgroup. Based upon this, eribulin was approved in for liposarcoma but not LMS [19].

SARC 028 study was a Phase II trial in which ten patients each of pleomorphic undifferentiated sarcoma, LMS, synovial sarcoma and liposarcoma (a total of 40 patients) were given pembrolizumab every 21 days [20]. There was no response in the LMS subgroup indicating that it might not be sensitive to immunotherapy. Similarly, in another Phase II trial evaluating single agent nivolumab, of 12 patients there was not even a single positive response [21]. Recently it has been shown that a small fraction of LMS patients might show $A L K$ gene rearrangement and might be sensitive to ALK inhibitors [22]. Though the advancements in the last few years have had mixed or only marginal success, with each and every trial, the understanding of the disease is definitely getting better.

\section{Conclusion \& future perspective}

In the present patient with gastric LMS, trabectedin demonstrated an excellent disease control despite being exposed to multiple lines of chemotherapy. The well-maintained quality of life and minimal toxicity profile is worth highlighting. However, we must keep looking for biomarkers in order to optimize treatment with these novel agents.

\section{Author contributions}

All authors contributed equally to the study design and methodology. S Rastogi, K Kalra, P Manasa wrote the first draft. V Mehta and M Rajawat edited the paper. S Rastogi, K Kalra and V Mehta revised the paper.

Financial \& competing interests disclosure

The authors have no relevant affiliations or financial involvement with any organization or entity with a financial interest in or financial conflict with the subject matter or materials discussed in the manuscript. This includes employment, consultancies, honoraria, stock ownership or options, expert testimony, grants or patents received or pending, or royalties.

No writing assistance was utilized in the production of this manuscript. 
Ethical conduct of research

The authors state that they have obtained appropriate institutional review board approval or have followed the principles outlined in the Declaration of Helsinki for all human or animal experimental investigations. In addition, for investigations involving human subjects, informed consent has been obtained from the participants involved.

\section{Open access}

This work is licensed under the Creative Commons Attribution 4.0 License. To view a copy of this license, visit http://creativecomm ons.org/licenses/by/4.0/

\section{Executive summary}

- Metastatic leiomyosarcoma (LMS) remains a disease with a dismal outlook, with an urgent need for new therapies. The last decade has seen major advancement in terms of collaborative studies with newer agents.

- Trabectedin is a novel therapeutic agent with a pleotropic mechanism of action approved after two lines of therapy in liposarcoma and LMS.

- Trabectadin is a relatively well tolerated chemotherapeutic agent with a noncumulative toxicity profile. Major toxicities are nausea, fatigue, neutropenia, thrombocytopenia, increased alanine amino transferase, vomiting and anemia.

- In our patient with metastatic gastric LMS, the disease was nonprogressive till last follow up after receiving 17 cycles of trabectedin with minimal toxicity.

- As this patient responded to combination of gemcitabine-docetaxel, pazopanib and trabectedin it is possible that the behaviour of gastric LMS is similar to extremity LMS.

- Future research looking for biomarkers to delineate the correct subtype of patient for each therapy holds the key for better patient selection.

\section{References}

Papers of special note have been highlighted as: $\bullet$ of interest; $\bullet \bullet$ of considerable interest

1. van der Graaf WT, Blay JY, Chawla SP et al. Pazopanib for metastatic soft-tissue sarcoma [PALETTE]: a randomised, double-blind, placebo-controlled Phase 3 trial. Lancet 379(9829), 1879-1886 (2012).

- This was the major landmark trial which demontrated the efficacy of pazopanib in metastatic leiomyosarcoma.

2. Demetri GD, von Mehren M, Jones RL et al. Efficacy and safety of trabectedin or dacarbazine for metastatic liposarcoma or leiomyosarcoma after failure of conventional chemotherapy: results of a Phase III randomized multicenter clinical trial. J. Clin. Oncol. 34(8), 786-793 (2016).

- This was also a landmark trial which showed the efficacy and safety of trabectedin and provided wonderful option in the treatment of metastatic liposarcomas and leiomyosarcoma.

3. D'Incalci M, Jimeno J. Preclinical and clinical results with the natural marine product ET-743. Expert Opin. Investig. Drugs. 12(11), 1843-1853 (2003).

4. Zewail-Foote M, Hurley LH. Ecteinascidin 743: a minor groove alkylator that bends DNA toward the major groove. J. Med. Chem. 42(14), 2493-2497 (1999).

5. Mehta V, Rajawat M, Rastogi S, Phulware RH, Mezencev R. Leiomyosarcoma of the stomach with metastasis to the liver: a case report with review of the literature. Future Sci. OA 4(2), FSO264 (2018).

6. Moody JS, Kozak KR. Gastric leiomyosarcomas: aSEER analysis. J. Clin. Oncol. 30(Suppl. 4), 106-106 (2012).

7. Leporini C, Patane M, Saullo F et al. A comprehensive safety evaluation of trabectedin and drug-drug interactions of trabectedin-based combinations. Biodrugs 28(6), 499-511 (2014).

8. Baldi GG, Di Donato S, Fargnoli R et al. Complete response after rechallenge with trabectedin in a patient with previously responding high-grade undifferentiated sarcoma. Anticancer Drugs 27(9), 908-1913 (2016).

9. Martin-Liberal J, Judson I. Safety evaluation of trabectedin in treatment of soft-tissue sarcomas. Expert Opin. Drug Saf. 12(6), 905-911 (2013).

10. Le Cesne A, Ray-Coquard I, Duffaud F et al. Trabectedin in patients with advanced soft tissue sarcoma: a retrospective national analysis of the French Sarcoma Group. Eur. J. Cancer 51(6), 742-750 (2015).

11. Tavella K, Villanucci A, Vannini L et al. Stable disease in a patient with metastatic leiomyosarcoma treated with trabectedin. Anticancer Drugs 28(4), 465-468 (2017).

12. Nteli VA, Knauf W, Janton-Klein A, El-Safadi S. Long-lasting response to trabectedin in a patient with metastatic uterine leiomyosarcoma: a case report. Case Rep. Oncol. 11(1), 81-89 (2018).

-. Similar case report showed prolong response of trabectedin in soft tissue sarcoma. 
13. Haslbauer F. Long-term progression-free survival in a patient with metastatic leiomyosarcoma of the inguinal region treated with trabectedin. Case Rep. Oncol. 11(1), 246-251 (2018).

-. Similar case report showed prolong response of trabectedin in soft tissue sarcoma.

14. Corrado G, Salutari V, Fuoco G, Lucidi A, Ferrandina G. Prolonged clinical response to trabectedin in a heavily pretreated patient with advanced uterine leiomyosarcoma: a case report and literature review. Gynecol. Oncol. 121(2), 416-417 (2011).

15. Lorusso D, Scambia G, Pignata S et al. Prospective Phase II trial of trabectedin in BRCA-mutated and/or BRCAness phenotype recurrent ovarian cancer patients: the MITO 15 trial. Ann. Oncol. 27(3), 487-493 (2016).

16. Tap WD, Jones RL, Van Tine BA et al. Olaratumab and doxorubicin versus doxorubicin alone for treatment of soft-tissue sarcoma: an open-label Phase $1 \mathrm{~b}$ and randomised phase 2 trial. Lancet. 388(10043), 488-497 (2016).

17. Tap WD, Wagner AJ, Papai $Z$ et al. ANNOUNCE: A randomized, placebo [PBO]-controlled, double-blind, Phase [Ph] III trial of doxorubicin [dox] + olaratumab versus dox $+\mathrm{PBO}$ in patients [pts] with advanced soft tissue sarcomas [STS]. J. Clin. Oncol. 37(Suppl. 18), LBA3-LBA (2019).

18. Schoffski P, Chawla S, Maki RG et al. Eribulin versus dacarbazine in previously treated patients with advanced liposarcoma or leiomyosarcoma: a randomised, open-label, multicentre, Phase 3 trial. Lancet. 387(10028), 1629-1637 (2016).

19. Rastogi S, Gupta V. Eribulin approval in advanced liposarcoma - successful drug or a weaker methodology? Ind. J. Med. Paediatr. Oncol. 38(1), 2-3 (2017).

20. Tawbi HA, Burgess M, Bolejack V et al. Pembrolizumab in advanced soft-tissue sarcoma and bone sarcoma [SARC028]: a multicentre, two-cohort, single-arm, open-label, Phase 2 trial. Lancet Oncol. 18(11), 1493-501 (2017).

21. Ben-Ami E, Barysauskas CM, Solomon S et al. Immunotherapy with single agent nivolumab for advanced leiomyosarcoma of the uterus: Results of a Phase 2 study. Cancer 123(17), 3285-3290 (2017).

22. Davis LE, Nusser KD, Przybyl J et al. Discovery and characterization of recurrent, targetable ALK fusions in leiomyosarcoma. Mol. Cancer Res. 17(3), 676-685 (2019). 